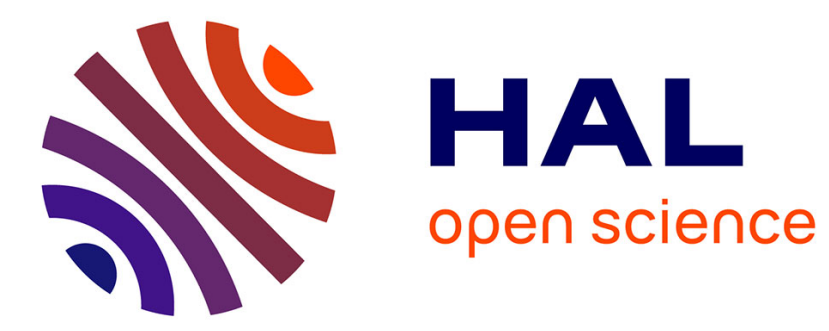

\title{
Resonant tunneling between two continua
}

L. Dobrzynski, Bahram Djafari-Rouhani, Abdellatif Akjouj, Jerome O. Vasseur, J. Zemmouri

\section{To cite this version:}

L. Dobrzynski, Bahram Djafari-Rouhani, Abdellatif Akjouj, Jerome O. Vasseur, J. Zemmouri. Resonant tunneling between two continua. Physical Review B, 1999, 60 (15), pp.10628-10631. 10.1103/PhysRevB.60.10628 . hal-03301332

\section{HAL Id: hal-03301332 https://hal.science/hal-03301332}

Submitted on 27 Jul 2021

HAL is a multi-disciplinary open access archive for the deposit and dissemination of scientific research documents, whether they are published or not. The documents may come from teaching and research institutions in France or abroad, or from public or private research centers.
L'archive ouverte pluridisciplinaire HAL, est destinée au dépôt et à la diffusion de documents scientifiques de niveau recherche, publiés ou non, émanant des établissements d'enseignement et de recherche français ou étrangers, des laboratoires publics ou privés. 


\title{
Resonant tunneling between two continua
}

\author{
L. Dobrzynski, B. Djafari-Rouhani, A. Akjouj, and J. O. Vasseur* \\ Laboratoire de Dynamique et Structures des Matériaux Moléculaires, UPRESA CNRS 8024, UFR de Physique, Université de Lille 1, \\ 59655 Villeneuve d'Ascq Cedex, France \\ J. Zemmouri \\ Laboratoire de Physique des Lasers, Atomes et Molécules, Centre d'Etudes et de Recherches Lasers et Applications, UMR CNRS 8523, \\ Université de Lille 1, 59655 Villeneuve d'Ascq Cedex, France \\ (Received 25 May 1999)
}

\begin{abstract}
We consider the tunneling between two monomode continua through a monomode structure with the symmetry of two orthogonal mirror planes. We derive in closed form the conditions for selective transfer of a single propagating state from one continuum to the other, leaving all other neighbor states unaffected. We illustrate the results of this analysis by analytical solutions for a simple structure made out of one-dimensional photonic waveguides. These theoretical results are confirmed by experiments using coaxial cables.

[S0163-1829(99)16735-3]
\end{abstract}

For the last ten years, complete channel drop tunneling between one-dimensional continua has been studied extensively for electrons ${ }^{1}$ as well as for electromagnetic waves. ${ }^{2,3}$ This selective transfer of propagating state from one continuum to the other, leaving the other neighbor states unaffected, may occur when the continua are coupled through a coupling element that supports localized resonant states. The understanding of channel drop tunneling between onedimensional continua is of fundamental and practical interest, as well as for monoenergy electrons as for singlefrequency photons. Applications of such transfer processes are important for wavelength demultiplexing in optical communications $^{4,5}$ and for electron spectroscopy. ${ }^{1}$ In a recent theoretical work, ${ }^{2}$ Fan et al. discussed the criteria for a complete transfer on the basis of symmetry arguments. They demonstrated the relevance of their analysis by numerical simulations, using a finite-difference time domain scheme, of the propagation of electromagnetic waves in a twodimensional defective photonic crystal.

In this paper, we pursue the appealing possibility of devising simple structures exhibiting resonant coupling at a specific frequency. We propose a model system and give in closed form conditions for complete channel drop tunneling for monomode coupling structures having the symmetry of two orthogonal mirror planes. We then illustrate the results of this analytical study on the example of an original structure made out of monomodes photonic waveguides. However the system model is general and may be easily transposed to other excitations such as electrons, magnons, or acoustic waves. Simple experiments using coaxial cables confirming the theoretical results for the above original structure are also reported.

Let us consider the generic system schematically presented in Fig. 1(a). This system is supposed to have the symmetry of two mirror planes. The two continua are the two infinite lines passing by, respectively, points $(1,2)$ and $(3,4)$. It is also convenient to consider the finite system $(1,2,3,4)$ obtained by removing the four semi-infinite lines at points $1,2,3$, and 4 . When a propagating state is excited in the line attached to point 1 , the corresponding reflection $R$ and transmission coefficients $T_{1 j}, j=2,3,4$ are easily found to be related to the elements of the Green's function of this system by the following relations:

$$
\begin{gathered}
R=|1+2 i \alpha g(11)|^{2}, \\
T_{1 j}=|2 i \alpha g(1 j)|^{2}, \quad j=2,3,4 .
\end{gathered}
$$

In Eqs. (1), the parameter $\alpha$ is defined, for monomode electromagnetic waves, as $\alpha=\omega \sqrt{\varepsilon} / c$, where $\omega$ is the frequency, $c$ the speed of light, $\varepsilon$ the relative dielectric constant of all
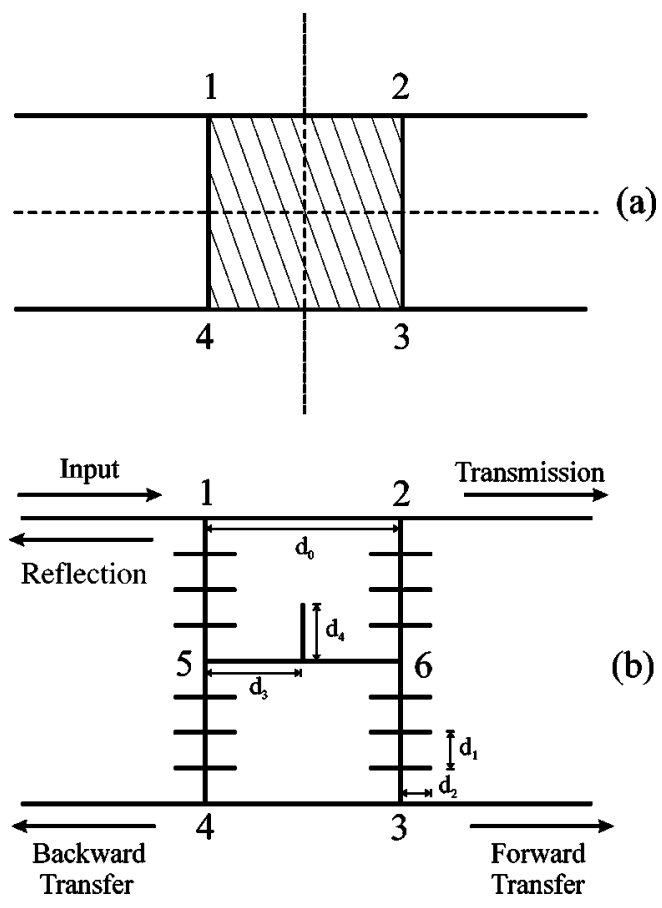

FIG. 1. (a) The general system under consideration. (b) The peculiar system for application. 
the photonic waveguides, and $i=\sqrt{-1}$. Taking into account the symmetry of the two mirror planes shown by dashed lines in Fig. 1(a), we obtain

$$
\begin{aligned}
& g(11)=Z_{1}+Z_{2}+Z_{3}+Z_{4}, \\
& g(12)=Z_{1}+Z_{2}-Z_{3}-Z_{4}, \\
& g(13)=Z_{1}-Z_{2}+Z_{3}-Z_{4}, \\
& g(14)=Z_{1}-Z_{2}-Z_{3}+Z_{4},
\end{aligned}
$$

where

$$
Z_{n}=1 /\left[4 \alpha\left(y_{n}-i\right)\right], \quad n=1,2,3,4 .
$$

In Eq. (3), the $y_{n}$ are purely real quantities determined by the finite structure contained in the shaded square $(1,2,3,4)$. One finds then that in order to have a complete transfer of a propagating state at a given frequency $\omega_{0}$ from site 1 to site 3 (namely, $T_{13}=1$ and $R=T_{12}=T_{14}=0$ ), one must fulfill the following conditions:

$$
y_{1}=y_{3}=-1 / y_{2}=-1 / y_{4} .
$$

If one wants this frequency $\omega_{0}$ to be in the middle of a frequency domain for which only direct transmission exists (namely, $T_{12}=1$ and $R=T_{13}=T_{14}=0$ ), one must fulfill in this domain the following conditions:

$$
y_{1}=y_{2}=-1 / y_{3}=-1 / y_{4} .
$$

In other words, these conditions (5) require that the system that couples the two continua must have a gap in the above-defined frequency domain. Conditions (4) imply that the complete system must have one resonant frequency $\omega_{0}$ inside this gap. These conclusions are equivalent to the ones obtained by Fan et al. ${ }^{2}$ for such a system. Conditions (4) and (5) enable us to determine completely from closed form expressions the geometrical parameters of the system.

In what follows, we illustrate these general results by choosing one very simple system, shown schematically in Fig. 1(b). This system is built from the two infinite monomode waveguides passing, respectively, by the points $(1,2)$ and $(3,4)$. The distance between 1 and 2 , called $d_{0}$, is the same as that between 3 and 4 . Four identical monomode photonic structures are branched between points $(1,5),(5,4)$, $(2,6)$, and $(6,3)$. These structures have $N$ equidistant (distance $d_{1}$ ) sites. Stars of $N^{\prime}$ side branches of length $d_{2}$ are grafted onto the $(N-2)$ internal sites. In Fig. 1(b), $N$ and $N^{\prime}$ are equal to 5 and 2, respectively. Such photonic structures were shown ${ }^{6}$ to have giant gaps. They enable us also to adjust the frequency range of these gaps to almost any desired domain by tuning the distance $d_{2}, d_{1}$ and the numbers $N$ and $N^{\prime}$. Between points 5 and 6 is fixed one waveguide of length $2 d_{3}$ with a side branch of length $d_{4}$ in its middle.

We give now the analytical expressions of the $y_{n}$ defined in Eq. (3). Let us define the quantities

$$
\begin{gathered}
A_{m}=-1 / \tan \left(\alpha d_{m}\right), \\
B_{m}=1 / \sin \left(\alpha d_{m}\right),
\end{gathered}
$$

with $m=0,1,2,3,4$.
$A_{0}$ and $B_{0}$ are associated with the finite parts of the infinite continua situated between points $(1,2)$ and $(3,4)$. We define also the terms $A_{5}$ and $B_{5}$ related to the structures with large gaps grafted between points $(1,5),(5,4),(2,6)$, and $(6,3)$ as

$$
A_{5}=-N^{\prime} A_{2}-A_{1}-B_{1} \sin \left(N k d_{1}\right) / \sin \left[(N-1) k d_{1}\right] \text {, }
$$

and

$$
B_{5}=B_{1} \sin \left(k d_{1}\right) / \sin \left[(N-1) k d_{1}\right],
$$

where $k$ is defined by

$$
\cos \left(k d_{1}\right)=-\frac{1}{B_{1}}\left(A_{1}+\frac{N^{\prime}}{2} A_{2}\right) .
$$

Expression (7c) is the dispersion relation of such an infinite star waveguide, when the electric field vanishes at the free extremities of the side branches.

The properties of the structure grafted between points 5 and 6 in Fig. 1(b) are related to

$$
B_{6}=-B_{3}^{2} /\left(2 A_{3}+A_{4}\right),
$$

and

$$
A_{6}=A_{3}+B_{6} .
$$

The definition of these quantities leads to the following expressions for the $y_{n}$ associated with the final system depicted in Fig. 1(b). They are given as

$$
y_{1}=y_{2}-2 B_{5}^{2} /\left(2 A_{5}+A_{6}+B_{6}\right),
$$

with

$$
\begin{gathered}
y_{2}=A_{0}+B_{0}+A_{5}, \\
y_{3}=A_{0}-B_{0}+A_{5}, \\
y_{4}=y_{3}-2 B_{5}^{2} /\left(2 A_{5}+A_{6}-B_{6}\right) .
\end{gathered}
$$

Now we are able to precise these system parameters for a complete channel drop tunneling between the two continua at a frequency $\omega_{0}$ falling in the middle of a given frequency band $\Delta \omega$. First the condition $y_{2} y_{3}=-1$ [Eqs. (4) and (5)] is satisfied for

$$
A_{5}\left(\omega_{0}\right)=0 .
$$

We determine the length $d_{0}$ such that

$$
y_{2}\left(\omega_{0}\right)=-y_{3}\left(\omega_{0}\right)=1 .
$$

by choosing

$$
\tan \left(\alpha_{0} d_{0} / 2\right)=1,
$$

where $\alpha_{0}=\omega_{0} \sqrt{\varepsilon} / c$.

So we study the quantity $A_{5}(\omega)$ given by Eq. (7a) and choose $N, N^{\prime}, d_{1}$, and $d_{2}$ in order that Eq. (10) shall be satisfied inside a gap corresponding to the required frequency band $\Delta \omega$. If $\Delta \omega$ is required to be even larger than the giant gaps reported for such star structures, one can improve on this point by using different dielectric constants in 


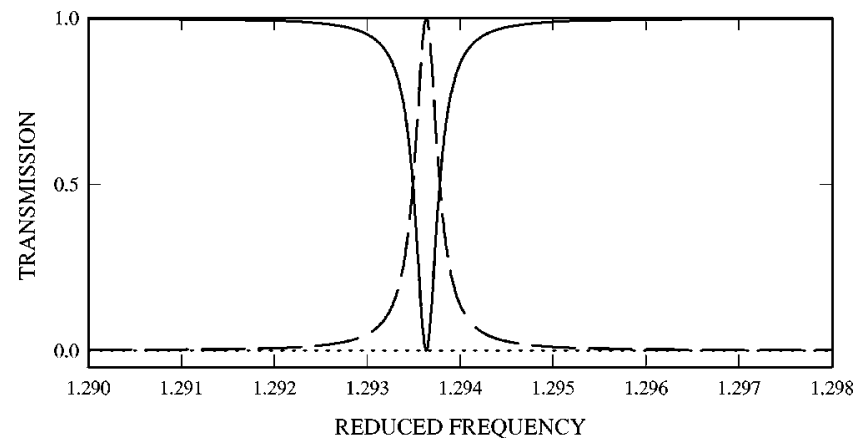

FIG. 2. Variation of the intensity of the transmitted signal from site 1 to site 2 (solid line), and of the forward signal ( $\left.T_{13}\right)$ (long dashed line), in the structure shown in Fig. 1(b) versus the reduced frequency. The dots represent the signal intensity in the backward direction $\left(T_{14}\right)$. These theoretical results were obtained for $N$ $=5, N^{\prime}=2, d_{0}=1.214189 d_{1}, d_{2}=0.4 d_{1}, d_{3}=1.2138 d_{1}$, and $d_{4}$ $=0.0003 d_{1}$. The resonant reduced frequency is $\Omega_{0}=1.293634$.

branches 1 and 2 and/or by putting two or more such structures in series. However, the quality factor of the transferred frequency peak increases when the gap $\Delta \omega$ increases, as well as the requirements on the precision on the distances $d_{m}$. The remainder of the conditions given by Eqs. (4) are then satisfied for

$$
A_{6}\left(\omega_{0}\right)=0
$$

and

$$
B_{6}\left(\omega_{0}\right)=B_{5}^{2}\left(\omega_{0}\right) \text {. }
$$

These last two conditions lead to

$$
\tan \left(\alpha_{0} d_{3}\right)=1 / B_{5}^{2}\left(\alpha_{0}\right),
$$

and

$$
\tan \left(\alpha_{0} d_{4}\right)=-\frac{1}{2} \tan \left(2 \alpha_{0} d_{3}\right),
$$

which define the distances $d_{3}$ and $d_{4}$ for a given $\omega_{0}$.

So, as announced above, our model system enables us to determine completely and in closed form the system parameters for a complete channel transfer once a frequency $\omega_{0}$ is chosen.

In order to illustrate the results of the above analytic theory, we present in Fig. 2, the variations of the transmission coefficients $T_{12}, T_{13}$, and $T_{14}$ versus the reduced frequency $\Omega=\omega \sqrt{\varepsilon} d_{1} / c$. Figure 2 shows together the dip (solid line) in the direct transmission from site 1 to site $2\left(T_{12}\right)$ and the forward drop (long dashed line) from site 1 to site 3 $\left(T_{13}\right)$. The backward transferred signal from site 1 to site 4 $\left(T_{14}\right)$ is completely absent over the entire frequency range and is represented by the dots in Fig. 2. This application was done for $N=5, N^{\prime}=2, d_{0}=1.214189 d_{1}, d_{2}=0.4 d_{1}, d_{3}$ $=1.2138 d_{1}$, and $d_{4}=0.0003 d_{1}$. The boundary condition at the free ends of all the side branches is $E=0$ (vanishing of the electric field). With these parameters, the reduced resonant frequency $\Omega$ equals 1.293634 . One can easily check that with these values, Eqs. (11) and (16) are verified. The quality factor of the sharp peaks defined as the ratio between the central frequency and the full width at half maximum is
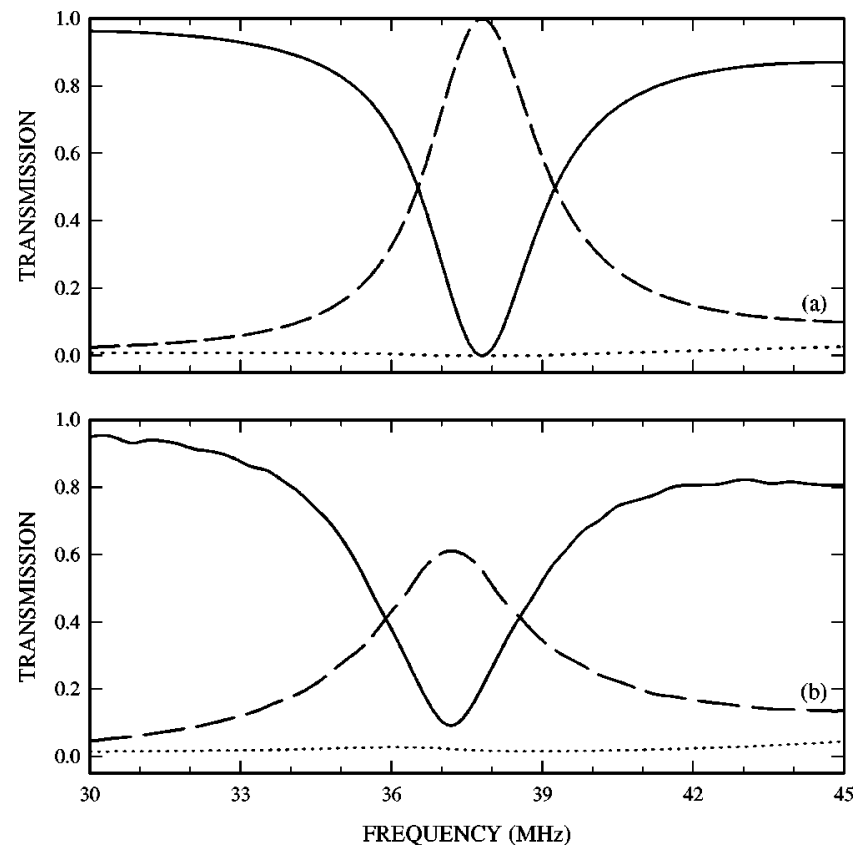

FIG. 3. Comparison between theoretical (a) and experimental (b) results for $\varepsilon=2.3, N=3, N^{\prime}=1, \quad d_{0}=1.3 m, d_{1}=1 \mathrm{~m}, d_{2}$ $=0.4 \mathrm{~m}, d_{3}=1.10 \mathrm{~m}$, and $d_{4}=0.15 \mathrm{~m}$. The boundary condition is $E$ $=0$ at the free ends of all the side branches. Solid line, direct transmission $\left(T_{12}\right)$; long dashed line, forward signal $\left(T_{13}\right)$; and dotted line, backward signal $\left(T_{14}\right)$.

of the order of 4300. A more complete study shows that this quality factor depends strongly on the characteristic lengths as well as on the integers $N$ and $N^{\prime}$.

In order to check the validity of the above theoretical model, we have examined, with a simple experimental setup, the behavior of the transmission coefficients in the frequency range of a few tens of $\mathrm{MHz}$. In these experiments, both the one-dimensional waveguides and the side branches are constituted by standard $50-\Omega$ coaxial cables and the transmission measurement is realized by using a tracking generator coupled to a spectrum analyzer. The top panel of Fig. 3 shows the theoretical variations of the transmission coefficients while the bottom panel presents the experimental measurements for the $E=0$ boundary condition and for $N$ $=3, N^{\prime}=1, \varepsilon=2.3, d_{0}=1.3 \mathrm{~m}, d_{1}=1 \mathrm{~m}, d_{2}=0.4 \mathrm{~m}, d_{3}$ $=1.10 \mathrm{~m}, d_{4}=0.15 \mathrm{~m}$. One notes that the behavior of the theoretical transmission coefficients in Figs. 2 and 3(a) is quite similar except for the weaker quality factor in Fig. 3(a). This is mainly explainable by the lower values of the integers $N$ and $N^{\prime}$ in Fig. 3(a) than in Fig. 2. The comparison between Figs. 3(a) and 3(b) reveals a good agreement between the theoretical predictions and the experimental spectra. Nevertheless the experimental resonant frequency is slightly different $(\approx 0.6 \mathrm{MHz})$ from the theoretical value of $37.8 \mathrm{MHz}$. An uncertainty on the length of the coaxial cables may explain this discrepancy. Another point of contention between experiment and theory stands in the amplitude of the peaks. For example, the experimental maximum of the forward signal drops to $62 \%$ compared to the $100 \%$ maximum obtained theoretically. This probably results from the attenuation of the signal occurring in the coaxial cables. ${ }^{6}$ It is also possible that one part of the input signal has been reflected at point 1 of the system [see Fig. 1(b)]. The reflection 
coefficient $R$ is strictly equal to zero in the theoretical calculation. An experimental measurement of this coefficient is not allowed with our experimental setup. One notes also that in the frequency range of Fig. 3, the intensity of the backward signal reaches a very low value. In spite of these slight disagreements, one can notice that our experimental measurements, made with a common experimental setup, validate fairly well the theoretical prediction. This is also, to our knowledge, the first experimental observation of the existence of resonant tunneling of electromagnetic waves.

In summary, we have investigated the tunneling between two monomodes continua coupled by a monomode structure. Inspired by the work of Fan et al., ${ }^{2}$ we have conceived a model system allowing a complete analytical and experimental study of the conditions for a selective transfer of propagating state from one continuum to the other leaving all the other neighbor states unaffected. Our theoretical model enables us to determine completely the geometric parameters of the monomode structure for a complete channel transfer at a specific frequency, once this frequency has been chosen. The frequency domain where the channel drop tunneling occurs only depends on the characteristic lengths of the constituents of the model system. Our theoretical model is then universal and valid in all the frequency domains of the electromagnetic spectrum. Moreover, the above formalism developed for electromagnetic waves can be easily transposed to electrons and magnons, as well as acoustic waves. This will be the subject of future work.

We think that our model system may have potential applications for making filtering or multiplexing devices. For example, a succession of a few "resonant tunneling structures" with different characteristics may allow us to extract several specific frequencies out of an input signal of large frequency band. Finally, one can notice that recent experimental works have shown that one-dimensional structures with dangling side branches can be fabricated at the submicrometer scale using high-resolution electron beam lithography. ${ }^{7,8}$ We hope then that the present work should lead to the manufacturing of optical devices having this geometry.

We are grateful to Professor M. Douay and Professor P. Niay, Laboratoire PHLAM, UFR de Physique, Université de Lille I for pointing out Refs. 4 and 5 and for helpful discussions. This work was partially supported by Le Conseil Régional Nord-Pas de Calais.
*Author to whom correspondence should be addressed.

${ }^{1}$ C. C. Eugster and J. A. del Alamo, Phys. Rev. Lett. 67, 3586 (1991), and references therein.

${ }^{2}$ S. Fan, P. R. Villeneuve, J. D. Joannopoulos, and H. A. Haus, Phys. Rev. Lett. 80, 960 (1998), and references therein.

${ }^{3}$ H. A. Haus and Y. Lai, J. Lightwave Technol. 10, 57 (1992).

${ }^{4}$ S. Berthuys, L. Lablonde, L. Rivoallan, J. F. Bayon, L. Brilland, and E. Delevaque, Electron. Lett. 34, 1250 (1998).

${ }^{5}$ T. T. Vu, R. J. Orazi, and M. N. McLandrich, Electron. Lett. 34, 583 (1998).
${ }^{6}$ L. Dobrzynski, A. Akjouj, B. Djafari-Rouhani, J. O. Vasseur, and J. Zemmouri, Phys. Rev. B 57, R9388 (1998).

${ }^{7}$ S. Foresi, P. R. Villeneuve, J. Ferrera, E. R. Thoen, G. Steinmeyer, S. Fan, J. D. Joannopoulos, L. C. Kimmerling, H. I. Smith, and E. P. Pippen, Nature (London) 390, 143 (1997).

${ }^{8}$ G. Feiertag, W. Ehrfeld, H. Freimuth, H. Kolle, H. Lehr, M. Schmidt, M. M. Sigalas, C. M. Soukoulis, G. Kiriakidis, T. Pedersen, J. Kuhl, and W. Koenig, Appl. Phys. Lett. 71, 1441 (1997). 